Journal of Animal and Veterinary Advances 11 (10): 1753-1756, 2012

ISSN: $1680-5593$

(C) Medwell Journals, 2012

\title{
Antioxidant Activity of the Water-Soluble and Alkali-Soluble Polysaccharides from Chinese Truffle Tuber sinense
}

\author{
${ }^{1,3}$ Ling Zhao, ${ }^{1,3}$ Kaiyu Wang, ${ }^{1}$ Ruqi Yang, ${ }^{2}$ Xun Wang, ${ }^{2}$ Defang Chen and ${ }^{1,3}$ Fei Shi \\ ${ }^{1}$ College of Verterinary Medicine, ${ }^{2}$ College of Animal Science and Technology, \\ ${ }^{3}$ Key Laboratory of Animal Disease and Human Health of Sichuan Province, \\ Sichuan Agricultural University, Ya'an, China
}

\begin{abstract}
To deeply explore the pharmacological activities of the Chinese truffle Tuber sinense (CT) in this study, three kinds of the crude polysaccharides from Chinese Truffle Tuber sinense (CTCP) were extracted by the method of water extracting-alcohol precipitating which include Water-soluble Polysaccharide (W-CTCP) and Alkali-soluble Polysaccharides (A-CTCPI, A-CTCPII) and then the polysaccharide content of the CTCP were determined. The antioxidant activities of the CTCPs were evaluated by reducing power, superoxide radical and hydroxyl radical free radical-scavenging assay. As the results, the polysaccharide (W-CTCP, A-CTCPI and A-CTCPII) content was $46.76,74.05$ and $57.68 \%$, respectively. The polysaccharides all exhibited antioxidant activities in vitro and $\mathrm{W}-\mathrm{CTCP}$ showed the strongest activity. It can be concluded that the polysaccharides extracted from CT should be explored as a potential natural antioxidant agent for use in functional foods or medicine.
\end{abstract}

Key words: Crude polysaccharide, Chinese truffle Tuber sinense, antioxidant, superoxide radical, medicine

\section{INTRODUCTION}

Truffle, the fruiting bodies of ascomycetous fungi belonging to the genus Tuber is found worldwide (Hawksworth et al., 1995). Truffles are hypogeous fungi which live in symbiosis with trees and some shrubs. Previous reports have showed that truffles of Saudi Arabia contained protein, amino acids, fat, crude fiber, ash and ascorbic acid, zinc, manganese and iron (Sawaya et al., 1985). Many ingredients from the fruiting-bodies of truffles have been isolated and they showed important biological activities (Gao et al., 2002; Hu et al., 1994).

Polysaccharide is one of the most important compounds in mushrooms. A number of reports have showed that polysaccharides have various biological activities such as antioxidant, anti-tumor, antiinflammatory and immunomodulation (Kim et al., 2008; Mizuno et al., 1996; Wu et al., 2010). Recent research has indicated that truffle polysaccharide extracted by water extracting exhibit antioxidant activity but there are no reports on antioxidant activity of truffle polysaccharides extracted by alkaline. In present study, three kinds of crude polysaccharides from Chinese truffle Tuber sinense were obtained by water-extracting and alkaline-extracting and then we evaluated the antioxidant activities of the
CTCPs by reducing power, superoxide radical and hydroxyl radical free radical-scavenging assay. Understanding of the antioxidant activities of the CTCPs would allow for development of a new natural antioxidant agent for use in functional foods or medicine.

\section{MATERIALS AND METHODS}

Extraction of the water-soluble and alkali-soluble polysaccharides from Chinese truffle Tuber sinense: The fruiting bodies of Chinese truffle Tuber sinense were obtained from a commercial market at Panzhihua, Sichuan province, China. The Traditional Water ExtractingAlcohol Precipitating Method was used to extract polysaccharides from the truffle. In brief, the fruiting bodies of the truffle were crushed into powder and dried at $60^{\circ} \mathrm{C}$. Then, the powder was extracted by $95 \%$ ethanol to remove the pigments, fat and inactivate enzymes. After filtering, the residues were air-dried and then boiling in distilled water for three times and $2 \mathrm{~h}$ for each time. By filtration, the debris was collected for the extraction of the Alkali-soluble Polysaccharide I (A-CTCPI) and the filtrate was concentrated in a rotary evaporator at $60^{\circ} \mathrm{C}$. Four volumes of ethanol were added to the extract and the mixture was left standing $12 \mathrm{~h}$. The precipitate was washed successively with ethanol and acetone then dried

Corresponding Author: Kaiyu Wang, Key Laboratory of Animal Disease and Human Health of Sichuan Province,

Sichuan Agricultural University, Ya'an, China 
at $50^{\circ} \mathrm{C}$ to obtain the Water-soluble Polysaccharide (W-CTCP). To extract A-CTCPI, the above-mentioned debris was dispersed for $12 \mathrm{~h}$ into $0.5 \mathrm{~mol} \mathrm{~L} \mathrm{~L}^{-1} \mathrm{NaOH}$ solution under stirring at room temperature. The extraction solution was filtered and the residues were collected for the extraction of the Alkali-soluble Polysaccharide II (A-CTCPII). The extract was neutralized with hydrochloric acid then was concentrated in a rotary evaporator at $60^{\circ} \mathrm{C}$. The remaining steps were the same as the preparation of W-CTCP. To extract A-CTCPП, residues collected from the extraction procedure of A-CTCPI were dispersed for $12 \mathrm{~h}$ into $1.0 \mathrm{~mol} \mathrm{~L}^{-1} \mathrm{NaOH}$ solution under stirring at room temperature. The extraction solution was filtered and the extract was neutralized with hydrochloric acid. The supernatant containing A-CTCPII was concentrated, ethanol precipitation and then dried. The total carbohydrate content of the water-soluble and alkalisoluble polysaccharides from Chinese truffle Tuber sinense was estimated by the Phenol-Sulfuric Acid Method (DuBois et al., 1956). The reducing sugar content was determined according to the method of Hodge and Hofreiter (Hodge and Hofreiter, 1962). The crude polysaccharide content was the subtraction of reducing sugar from total carbohydrates.

Hydroxyl radical-scavenging assay: The hydroxyl radicalscavenging activity was measured using the method reported by Liu et al. (2010) with slight modification. Hydroxyl radicals were generated by Fenton reaction in the system of $\mathrm{FeSO}_{4}$ and $\mathrm{H}_{2} \mathrm{O}_{2}$. The reaction mixture that contained polysaccharides $1 \mathrm{~mL}$ was incubated with phenanthroline $(7.5 \mathrm{mM}$ and $1 \mathrm{~mL})$, distilled water $(1 \mathrm{~mL})$, ferrous sulfate $(0.75 \mathrm{mM}$ and $1 \mathrm{~mL})$ and hydrogen peroxide $(0.01 \%$ and $1 \mathrm{~mL})$ in phosphate buffer ( $20 \mathrm{mM}$ and $\mathrm{pH} \mathrm{7.4)} \mathrm{for} 30 \mathrm{~min}$ at $37^{\circ} \mathrm{C}$ and the absorbance was read at $510 \mathrm{~nm}$. The scavenging activity of hydroxyl radical was expressed using the following equation:

Hydroxyl radical-Scavenging activity $(\%)=\frac{A_{s}-A_{1}}{A_{0}-A_{1}} \times 100$

Where:

$\mathrm{A}_{s}=$ Absorbance of the sample

$\mathrm{A}_{1}=$ Absorbance of the control solution containing 1,10-phenanthroline, $\mathrm{FeSO}_{4}$ and $\mathrm{H}_{2} \mathrm{O}_{2}$

$\mathrm{A}_{0}=$ Absorbance of the blank solution containing 1,10-phenanthroline and $\mathrm{FeSO}_{4}$

Superoxide radical-scavenging assay: The scavenging effect on superoxide radicals was determined according to the method of Li et al. (2011) and Ding et al. (2010). Briefly, $4.5 \mathrm{~mL}$ Tris-HCl buffer ( $50 \mathrm{mM}$ and $\mathrm{pH} 8.2), 1 \mathrm{~mL}$ polysaccharide solution were mixed and incubated at $25^{\circ} \mathrm{C}$ for $20 \mathrm{~min}$ and then $0.2 \mathrm{~mL}$ and $3 \mathrm{mM}$ pyrogallol solution was added to initiate the reaction the change speed of absorbance $(\mathrm{A} / \mathrm{min})$ of the reactive solution was measured at $325 \mathrm{~nm}$ against the blank. The capability of scavenging superoxide radical was calculated using the following equation:

Superoxide radical-Scavenging activity $(\%)=\frac{\mathrm{V}_{0}-\mathrm{V}_{1}}{\mathrm{~V}_{0}} \times 100$

Where:

$\mathrm{V}_{0}=$ The change speed of absorbance of the control group in the superoxide radical generation system

$\mathrm{V}_{1}=$ The change speed of absorbance of the test sample

Reducing power: The reducing power was evaluated according to the method of Deng et al. (2011). In brief, $1 \mathrm{~mL}$ polysaccharides solutions at different concentration was mixed with $2.5 \mathrm{~mL}$ phosphate buffer $\left(0.02 \mathrm{~mol} \mathrm{~mL}^{-1}, \mathrm{pH} 6.6\right)$ and $2.5 \mathrm{~mL}$ of ferricyanide solution $\left(10 \mathrm{~g} \mathrm{~L}^{-1}\right)$. The mixture was incubated at $50^{\circ} \mathrm{C}$ for $20 \mathrm{~min}$ and then $2.5 \mathrm{~mL}$ trichloroacetic acid solution $\left(100 \mathrm{~g} \mathrm{~L}^{-1}\right)$ $2.5 \mathrm{~mL}$ distilled water and $0.5 \mathrm{~mL}$ ferric chloride solution (0.5 ML, $1 \mathrm{~g} \mathrm{~L}^{-1}$ ) was added. The absorbance of the mixture was read at $700 \mathrm{~nm}$.

\section{RESULTS AND DISCUSSION}

Extraction of crude polysaccharides from Chinese truffle Tuber sinense and content analysis: The water-soluble and alkali-soluble polysaccharides from Chinese truffle Tuber sinense were extracted by water and alkaline. As shown in Table 1 the contents of the polysaccharides in W-CTCP, A-CTCP I and A-CTCP II were determined as $46.76,74.05$ and $57.68 \%$, respectively. A-CTCP I showed the highest polysaccharide content while W-CTCP showed the highest reducing sugar content.

Hydroxyl radical-scavenging activity: As shown in Fig. 1 W-CTCP was found to have stronger scavenging activity of hydroxyl radical than A-CTCP I and A-CTCP II. W-CTCP exhibited hydroxyl radicals scavenging activity in a concentration-dependent manner. Scavenging effects of W-CTCP were $13.71 \sim 83.92 \%$ at concentration of 0.1-2.4 $\mathrm{mg} \mathrm{mL}^{-1}$. A-CTCP I and A-CTCP $\Pi$ showed a weak

Table 1: Determination of the content of polysaccharides from Chinese truffle Tuber sinense

\begin{tabular}{lccc}
\hline Sample name (\%) & W-CTCP & A-CTCP I & A-CTCP II \\
\hline Reducing sugar content & 5.73 & 0.00 & 0.00 \\
Polysaccharide content & 46.76 & 74.05 & 57.68 \\
The total sugar content & 52.49 & 74.05 & 57.68 \\
\hline
\end{tabular}




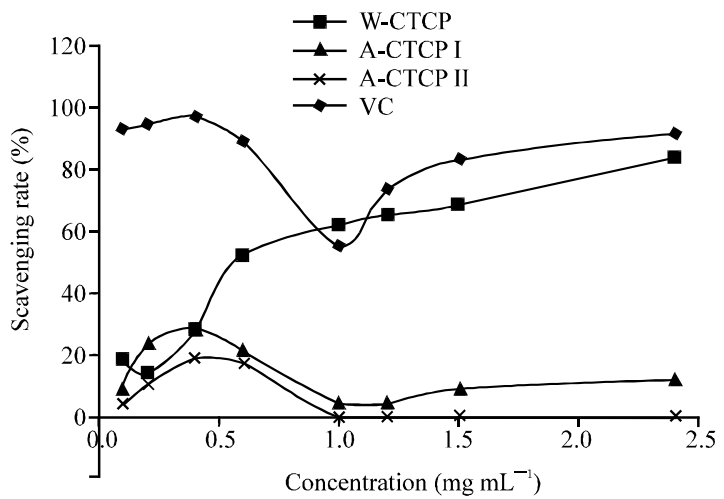

Fig. 1: Hydroxyl radicals scavenging activity of polysaccharide from $\mathrm{CT}$

scavenging ability and both of them have higher scavenging activities on hydroxyl radical at low concentration $\left(<1 \mathrm{mg} \mathrm{mL}^{-1}\right)$ than at higher concentration (1 2.4 $\mathrm{mg} \mathrm{mL}^{-1}$ ).

Superoxide radicals scavenging activity: The three polysaccharides were found to have the ability to scavenge superoxide radical at concentration between 0 and $2.4 \mathrm{mg} \mathrm{mL}^{-1}$ (Fig. 2). These results indicated that the water-extracting polysaccharide had a stronger superoxide radical-scavenging activity than the alkali-extracting polysaccharides. The superoxide radicals scavenging effects of the polysaccharides increased with increasing concentration. W-CTCP showed good superoxide anion scavenging activities $(45.9 \%)$ at the concentration of $2.4 \mathrm{mg} \mathrm{mL}^{-1}$ while A-CTCP I and A-CTCP II were only 13.5 and $35.98 \%$ at the same concentration, respectively.

Reducing power: A higher absorbance of the reaction mixture indicated greater reducing power. As shown in Fig. 3, the reducing power of the polysaccharides increased with increasing concentration between 0 and $0.6 \mathrm{mg} \mathrm{mL}^{-1}$. Though the reducing power of all samples was low in the test concentration, the water-extracting polysaccharide had a better reducing power than alkaliextracting polysaccharides at the same concentration. The reducing power of W-CTCP, A-CTCP I and A-CTCP II at $0.6 \mathrm{mg} \mathrm{mL}^{-1}$ were $0.332,0.07$ and 0.078 , respectively which were much weaker than those of ascorbic acid.

The oxidative damage, associated with reactive oxygen species is believed to be involved in many illnesses such as diabetes mellitus, arterisclerosis and cancer (Halliwell and Gutteridge, 1984). It is important to suppress the reactive oxygen species for treatment of the illness listed above. Polysaccharides as one of the most

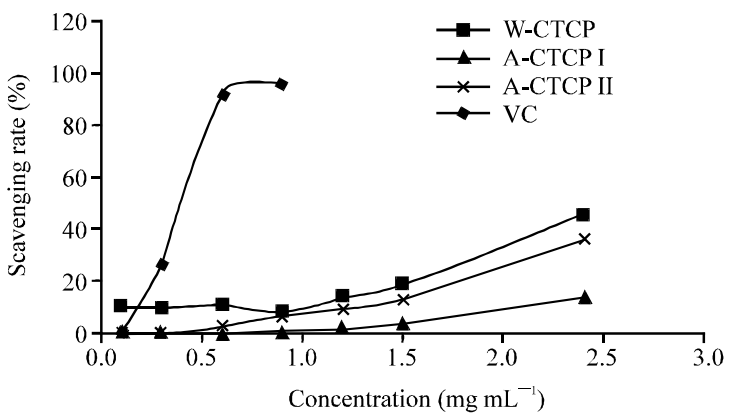

Fig. 2: Superoxide radicals scavenging activity of polysaccharide from $\mathrm{CT}$

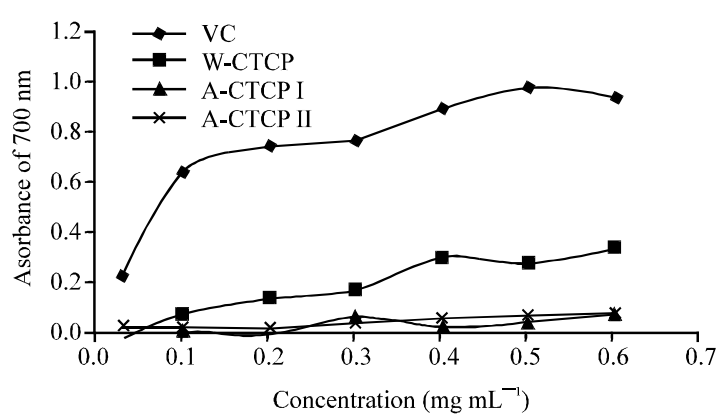

Fig. 3: Reducing power of polysaccharides from CT

important components of mushroom have been reported to have various bioactivies such as antioxidant, antitumor and immunodulatory activites (Li et al., 2011; Kim et al., 2008; Mizuno et al., 1996). For exmaple, the polysaccharides from Ganoderma atrum, Dictyophora indusiata, Grifola frondosa, Hericium erinaceus and Tricholoma giganteum, Tricholoma matsutake exhibited the antioxidant activity (Mau et al., 2002; Ding et al., 2010; Chen et al., 2008). In present study, three kinds of polysaccharides from Chinese truffle Tuber sinense were extracted by water and alkaline and several in vitro assays were applied to evaluate the antioxidant potential of the polysaccharides. W-CTCP exhibited excellent antioxidant activities and it is consistent with the reports of Jin-Zhong et al. (2011). Alkaline extracting polysaccharides also exhibited antioxidant acitivity but compared with W-CTCP, A-CTCP I and A-CTCP II had weaker antioxidant activities. The differences in antioxidant activity may be due to different structure and components. Meanwhile, the results also suggested that the $\mathrm{pH}$ of extraction solvent played an important role on bioactivity of polysaccharides. But the exact mechanism of the antioxidant activity of the polysaccharides from truffle has not been clarified and need further research. 


\section{CONCLUSION}

This study shows that three kinds of polysaccharides from Chinese truffle all exhibited antioxidant activity in vitro and W-CTCP have highest antioxidant activity among them. Polysaccharides of Chinese truffle could be explored as a potential natural antioxidant agent for use in functional foods or medicine.

\section{ACKNOWLEDGEMENTS}

This research was supported by the Department of Science and Technology of Sichuan province of China (No.: 09ZA083) and the Program for Changjiang Scholars and Innovative Research Team in University (No.: IRT0848).

\section{REFERENCES}

Chen, Y., M.Y. Xie, S.P. Nie, C. Li and Y.X. Wang, 2008. Purification, composition analysis and antioxidant activity of a polysaccharide from the fruiting bodies of Ganoderma atrum. Food Chem., 107: 231-241.

Deng, P., G. Zhang, B. Zhou, R. Lin and L. Jia et al., 2011. Extraction and in vitro antioxidant activity of intracellular polysaccharide by Pholiota adiposa SX-02. J. Biosci. Bioeng., 111: 50-54.

Ding, X., J. Tang, M. Cao, C.X. Guo and X. Zhang et al., 2010. Structure elucidation and antioxidant activity of a novel polysaccharide isolated from Tricholoma matsutake. Int. J. Biol. Macromol., 47: 271-275.

DuBois, M., K.A. Gilles, J.K. Hamilton, P.A. Rebers and F. Smith, 1956. Colorimetric method for determination of sugars and related substances. Anal. Chem., 28: 350-356.

Gao, J.M., A.L. Zhang, C.Y. Wang, X.H. Wang and J.K. Liu, 2002. A new ceramide from the ascomycete Tuber indicum. Chin. Chem. Lett., 13: 325-326.

Halliwell, B. and J.M. Gutteridge, 1984. Oxygen toxicity, oxygen radicals, transition metals and disease. Biochem. J., 219: 1-14.

Hawksworth, D.L., P.M. Kirk, B.C. Sutton and D.N. Pegler, 1995. Truffle: Dictionary of the Fungi. Cambridge University Press, Cambridge, UK., pp: 467-469.
Hodge, J.E. and B.T. Hofreiter, 1962. Determination of Reducing Sugars and Carbohydrates. In: Methods in Carbo-hydrate Chemistry, Whistler, R.L., M.L. Wolfrom, J.N. Be-Miller and F. Shafizadeh (Eds.). Academic Press Inc., New York, pp: 380-394.

$\mathrm{Hu}$ H., L. Peizhen, L. Tao, H. Bingqian and G. Yaowei, 1994. Effects of polysaccharide of tuber sinica on tumor and immune system of mice. J. Chin. Pharm. Univ., 125: 289-292.

Jin-Zhong, C., W. Li, S. Hong, F. Li and L. Yu, 2011. Study on extraction and anti-oxidant activity of crude olysaccharides from tuber indicum. J. Shanxi Univ., 34: 137-142.

Kim, J.Y., S.E. Byeon, Y.G. Lee, J.Y. Lee, J. Park, E.K. Hong and J.Y. Cho, 2008. Immunostimulatory activities of polysaccharides from liquid culture of pinemushroom Tricholoma matsutake. J. Microbiol. Biotechnol., 18: 95-103.

Li, H., J. Xu, Y. Liu, S. Ai, F. Qin, Z. Li, H. Zhang and Z. Huang, 2011. Antioxidant and moistureretention activities of the polysaccharide from Nostoc commune. Carbohydr. Polym., 83: 1821-1827.

Liu, Q.M., X.M. Yang, L. Zhang and G. Majetich, 2010. Optimization of ultrasonic-assisted extraction of chlorogenic acid from Folium eucommiae and evaluation of its antioxidant activity. J. Med. Plants Res., 4: 2503-2511.

Mau, J.L., H.C. Lin and S.F. Song, 2002. Antioxidant properties of several specialty mushrooms. Food. Res. Int., 35: 519-526.

Mizuno, T., P. Yeohlui, T. Kinoshita, C. Zhuang and H. Ito, 1996. Antitumor activity and chemical modification of polysaccharides from niohshimeji mushroom, Tricholma giganteum. Biosci. Biotechnol. Biochem., 60: 30-33.

Sawaya, W.N., A. Al-Shalhat, A. Al-Sogair and M. Al-Mohammad, 1985. chemical composition and nutritive value of truffles of Saudi Arabia. J. Food Sci., 50: 450-453.

Wu, D.M., W.Q. Duan, Y. Liu and Y. Cen, 2010. Antiinflammatory effect of the polysaccharides of golden needle mushroom in burned rats. Int. J. Biol. Macromol., 46: 100-103. 